\title{
Dynamic modification strategy of the Israeli carrier screening protocol: inclusion of the Oriental Jewish Group to the cystic fibrosis panel
}

\author{
Orit Reish, $M D^{1,2}$, Zvi U. Borochowitz, $M D^{3}$, Vardit Adir, PhD ${ }^{3}$, Mordechai Shohat, $M D^{2,4}$, \\ Mazal Karpati, PhD ${ }^{2,5}$, Atalia Shtorch, $P h D^{6}$, Avi Orr-Urtreger, $M D, P h D^{2,7}$, \\ Yuval Yaron, $M D^{2,7}$, Stavit Shalev, $M D^{8}$, Fuad Fares, $P h D^{9}$, Ruth Gershoni-Baruch, $M D^{10}$, \\ Tzipora C. Falik-Zaccai, $M D^{11}$, and Daphne Chapman-Shimshoni, PhD ${ }^{1}$
}

\begin{abstract}
Purpose: A retrospective population study was conducted to determine the carrier frequencies of recently identified mutations in Oriental Jewish cystic fibrosis patients. Methods: Data were collected from 10 medical centers that screened the following mutations: two splice site mutations-3121-1G $>$ A and $2751+1$ ins - and one nonsense mutation - the Y1092X in Iraqi Jews. One missense mutation, I1234V, was screened in Yemenite Jews. Results: A total of 2499 Iraqi Jews were tested for one, two, or all three mutations. The 3121-1G>A, Y1092X, and $2751+1$ ins T mutations had a carrier frequency of 1:68.5, 1:435, and 0 , respectively. In 1435 Yemenite Jews screened, I1234V had a carrier frequency of 1:130. Conclusion: The $0.84 \%$ allele frequency of the three Iraqi founder mutations falls within the Israeli Society of Medical Geneticists' inclusion criteria for screening of 1:60 carrier frequency; hence, Iraqi Jews were added to the carrier screening policy with a panel including the three Iraqi founder mutations in addition to the five Ashkenazi mutations previously detected in Eastern Jews. 2751 +1 insT that was detected in patients only was included in the screening panel to increase the detection rate. I1234V does not meet the inclusion criteria but is now offered on a diagnostic basis and can be added to the screening panel for individuals whose mixed origin includes Yemenite, in addition to protocol-recommended origins. This study demonstrates the dynamic modifications of the Israeli carrier cystic fibrosis screening protocol based on newly detected founder mutations in a large cohort, taking into account mutation impact and intercommunal admixture. Genet Med 2009:11(2):101-103.
\end{abstract}

Key Words: cystic fibrosis, carrier screening, Oriental Jews, population study

\footnotetext{
From the ${ }^{1}$ Genetics Institute, Assaf Harofeh Medical Center, Zerifin, Israel; ${ }^{2}$ Sackler School of Medicine, Tel Aviv University, Tel Aviv, Israel; ${ }^{3}$ The Simon Winter Institute for Human Genetics, Bnei-Zion Medical Center, Technion-Rappaport Faculty of Medicine, Haifa, Israel; ${ }^{4}$ Genetics Institute, Rabin Medical Center, Petah Tikva, Israel; ${ }^{5}$ Danek Gertner Institute of Human Genetics, Sheba Medical Center, Tel-Hashomer, Israel; ${ }^{6}$ Laboratory of Molecular Genetics, Sapir Medical Center, Kfar Saba, Israel; ${ }^{7}$ Genetics Institute, Tel Aviv-Sourasky Medical Center, Tel Aviv, Israel; ${ }^{8}$ Genetics Institute, Ha'emek Hospital, Afula, Israel; ${ }^{9}$ Department of Molecular Genetics, Carmel Medical Center, University of Haifa, Haifa, Israel; ${ }^{10}$ Genetics Institute, Rambam Medical Center, Haifa, Israel; and ${ }^{11}$ Genetics Institute, Western Galilee Hospital, Nahariya, Israel.
}

Orit Reish, MD, ABMG, Genetics Institute, Assaf Harofeh Medical Center, Zerifin 70300, Israel. E-mail: oreish@post.tau.ac.il

Disclosure: The authors declare no conflict of interest.

Submitted for publication July 13, 2008.

Accepted for publication September 23, 2008

DOI: $10.1097 /$ GIM.0b013e31818efd59
$T_{s}^{\mathrm{s}}$ he different Jewish ethnic groups were isolated from the surrounding non-Jewish populations as a result of religious restrictions and cultural differences, and from each other by geographical boundaries. ${ }^{1-3}$ Thus, their respective genetic loads differ, except for more ancient founder mutations common in all Jews that were acquired before the communities dispersed. ${ }^{2}$ With the establishment of the State of Israel in 1948, many of these Jews migrated back to Israel. Both the mutations characteristic to the specific ethnic groups and the common Jewish mutations created the basis for the ethnic-specific screens offered to the different communities in Israel.

As expected, the incidence and distribution of mutations of cystic fibrosis (CF) vary between the Jewish ethnic communities. ${ }^{4}$ A carrier CF screening was introduced in Israel once the major mutations in the various ethnic groups were identified. ${ }^{4,5}$ This screen was possible for the Ashkenazi, North African, Sephardic and Georgian Jews, for whom the tested mutations gave a high detection rate. ${ }^{4}$ As carrier frequencies are high in these groups, screening was provided to couples from the same or mixed origins. The population study described here was initiated to determine the carrier frequencies of recently identified mutations in CF patients of Oriental origin. ${ }^{6}$ It is based on data collected from genetics laboratories at 10 Israeli medical centers.

\section{MATERIALS AND METHODS}

\section{Participating centers}

Data were collected from 10 medical centers that performed carrier screening for CF: Assaf Harofeh (Zerifin), Bnei Zion (Haifa), Rabin (Petach Tikva), Sheba (Tel-Hashomer), Sapir (Kfar Saba), Tel Aviv (Tel Aviv), Haemek (Afula), Carmel (Haifa), Rambam (Haifa), and Western Galilee (Naharia). This retrospective study relates to samples collected and analyzed during the years 2005-2006.

\section{Subjects}

Data related to four Oriental mutations were collected from a total of 2499 individuals of homogeneous or mixed Iraqi origin, and 1435 individuals of homogeneous or mixed Yemenite origin. Definition of origin was based on individuals' reports at the time of blood collection. Participants were healthy individuals who performed the screening tests in a family planning context; therefore, some of the subjects were pregnant women. Individuals with signs of CF, or family history of CF were excluded from the study. Informed consent was obtained from all participants and the study was approved by the Institutional Review Board. 
Table 1 Primer sequences and restriction enzymes used for mutation analysis

\begin{tabular}{lcc}
\hline Mutations & \multicolumn{1}{c}{ Primers } & $\begin{array}{c}\text { Restriction } \\
\text { enzyme }\end{array}$ \\
\hline 3121-G $>$ A & CF-23 TCACTGACACACTTTGTCCA & MfeI \\
& CF-24 GAATCTCAAATAGCTCTTA & \\
& TAGC \\
Y1092X & CF-14 GCTCTGAATTTACATACTGCCAC & RsaI \\
& CF-15 ATAACCTATAGAAT \\
GCAGCA & \\
2751+1insT CF-25 ACAATGGTGGCATGAAACTG & RsaI \\
& CF-26 GTAATACTTTACAATAGAACAT & \\
I1234V & TCGTA & \\
& CF-21 CTCGAAAGTATGATTATTGA & DdeI \\
\hline
\end{tabular}

\section{Genetic analysis}

The Oriental Jewish mutations, 3121-1G > A, Y1092X, and $2751+1$ ins T, were tested on samples from Iraqi Jews, and the I1234V mutation was tested on Yemenite Jews. Genetic analysis consisted of polymerase chain reaction amplification followed by restriction enzyme analysis, ${ }^{5}$ using primers and enzymes listed in Table 1. For each mutation, the number of tested individuals and carriers from each origin was reported. The total number of alleles was calculated as follows: when origin was homogeneous, by multiplying the number of screened individuals by two (for two alleles per one subject); when origin was mixed, by multiplying the number of screened individuals by only the fraction of the origin under consideration.

\section{RESULTS}

The three founder Oriental Jewish mutations, Y1092X, 3121$1 \mathrm{G}>\mathrm{A}$, and $2751+1$ insT, were tested on 3474, 890, and 992 screened alleles of Jewish Iraqi origin, respectively; the I1234V mutation was tested on 2072 screened alleles of Yemenite origin (Table 2). In the absence of consensus on the mutation repertoire of the Oriental Jewish groups before this study, the number of mutations examined by each of the participating centers varied. The different numbers of total tested alleles for each mutation accounts for the different denominators in Table 2.
In the Iraqi Jewish population, the $3121-1 \mathrm{G}>\mathrm{A}$ mutation was the most frequent, with a carrier frequency of 1:68.5. The Y1092X mutation had a lower carrier frequency of 1:435, and the $2751+1$ insT mutation was not detected at all. In the Yemenite Jewish population, the I1234V mutation had a carrier frequency of $1: 130$.

\section{DISCUSSION}

A unique mutation spectrum for $\mathrm{CF}$ had been found previously in each of the Jewish communities, and for each community the detection rate differed. Five mutations provide at least a $95 \%$ detection rate among Ashkenazi Jewish CF patients, ${ }^{4}$ and additional specific mutations were found among Tunisian, Libyan, Moroccan, Turkish, and Georgian Jews, giving higher than $85 \%$ detection rates in these populations. ${ }^{4,6}$ The disease frequency in these communities is similar to that of the non-Jewish white frequency $(1: 2,400-1: 3,300)$. $^{7}$

The high incidence and rate of detection of the major mutations among these specific Jewish communities prompted the introduction of a carrier screening program in Israel in 1995,4,8 according to the policy established by the Israeli Society of Medical Geneticists (ISMG). This initial policy recommended screening Ashkenazi Jews for their five founder mutations, and adding the mutations specific to the non-Ashkenazi Jewish group to the screen. For example, for Tunisian Jews the $405+$ $1 \mathrm{G}>\mathrm{A}$ mutation was added to the five Ashkenazi mutations, and for Moroccan Jews the S549R and D1152H mutations were added to the five Ashkenazi mutations. ${ }^{8}$ Because approximately $25 \%$ of all marriages in Israel is intercommunal, ${ }^{9}$ changing the population admixture, the ISMG simplified the carrier protocol of CF screening in the year 2002: all non-Ashkenazi Jews were grouped together (not including the Oriental Jews because of lack of information) and were screened for a total of nine mutations - the five Ashkenazi and the four known non-Ashkenazi mutations $(405+1 \mathrm{G}>$ A S549R, W1089X, G85E). Jews of Georgian origin were tested for the five Ashkenazi mutations and the typical Georgian mutation (T360K). Accumulating information led to removal of $1717-1 \mathrm{G}>\mathrm{A}$ from the carrier screen because of its rarity, ${ }^{8}$ and $\mathrm{D} 1152 \mathrm{H}$ because of the mild phenotype. ${ }^{10}$ To increase the detection rate, when a carrier was detected in the screen, his or her partner was evaluated for at least those nine mutations, even if of Ashkenazi or Georgian origin.

Table 2 Number of mutant alleles and carriers in Iraqi and Yemenite Jewish populations

\begin{tabular}{|c|c|c|c|c|}
\hline Origin & Mutation & Allele frequency ${ }^{a}(\% ; 95 \% \mathrm{CI})$ & Carriers in population ${ }^{a}(\%)$ & Patients' alleles ${ }^{b}$ \\
\hline \multicolumn{5}{|l|}{ Iraq } \\
\hline \multicolumn{2}{|l|}{ Y1092X } & $4 / 3474(0.11 ; 0.03-0.24)$ & $1 / 435(0.23)$ & $3 / 8$ \\
\hline \multicolumn{2}{|l|}{$3121-1 \mathrm{G}>\mathrm{A}$} & $6.5 / 890(0.73 ; 0.25-1.46)$ & $1 / 68.5(1.46)$ & $1 / 3^{c}$ \\
\hline \multicolumn{2}{|l|}{$2751+1$ ins $\mathrm{T}$} & $0 / 992(0)$ & $0 / 496(0)$ & $2 / 8$ \\
\hline \multicolumn{5}{|l|}{ Yemen } \\
\hline \multicolumn{2}{|l|}{$\mathrm{I} 1234 \mathrm{~V}$} & $8 / 2072(0.39 ; 0.18-0.73)$ & $1 / 130(0.76)$ & $2 / 2^{d}$ \\
\hline $\begin{array}{l}{ }^{a} \text { Data refer to pre } \\
{ }^{b} \text { Data derived fro } \\
{ }^{c} \text { Refers to patient } \\
{ }^{d} \text { Two patients wh } \\
{ }^{\circ} \text {, denotes numb }\end{array}$ & $\begin{array}{l}\text { study. } \\
\text { uint et al. }{ }^{6} \\
\text { Kurdish Jew } \\
\text { ch had one Y } \\
\text { mutant allel }\end{array}$ & $\begin{array}{l}\text { sh parent and one of another origin. } \\
\text { total number of alleles tested; CI, der }\end{array}$ & iisher' & \\
\hline
\end{tabular}


The low frequency and lack of appropriate characterization of the common mutations in Oriental Jews ${ }^{4}$ precluded their inclusion in the screening protocol until 2005 when several mutations were identified among CF patients of Oriental origin. ${ }^{6}$ Three Iraqi Jewish alleles of eight studied carried the Y1092X, a premature termination mutation; two alleles carried the $2751+1$ insT, a donor splice site mutation; and another splice site mutation, 3121-1G > A, was detected in one allele from a Kurdish Jewish patient and in one allele from an Egyptian Jewish patient (CF consortium database). In that same study, ${ }^{6}$ two patients each having one Yemenite Jewish allele carried the I1234V mutation, whereas the second mutation was [Delta]F508 in one patient and W1282X in the other. Detection of these new founder Oriental mutations led, in 2005, to unofficially offering carrier testing to Jews of Iraqi and Yemenite origins in 10 genetics centers in Israel. The data gathered in 2006 and presented here assessed the distribution of these mutations among a large population, aimed at providing evidence on which to update the official ISMG policy. The data revealed that $3121-1 \mathrm{G}>\mathrm{A}, \mathrm{Y} 1092 \mathrm{X}$, and $2751+1$ insT mutations had carrier frequencies of 1:68.5, 1:435, and 0 (allele frequency $0.73,0.11,0 \%$ ), respectively, among the Iraqi Jews, adding up to a frequency of $0.84 \%$. This allele frequency fell within the ISMG inclusion criteria of 1:60 carrier frequency and a severe disease impact, leading to the official inclusion of Iraqi Jews in the carrier screen. Inclusion of the $3121-1 \mathrm{G}>\mathrm{A}$ and the Y1092X mutations in the panel is also within the suggested criteria of $\geq 0.1 \%$ allele frequency used for the pan-ethnic United States mutation panel. ${ }^{11,12}$ Although the $2751+1$ insT mutation was not detected among individuals in this study, it was found in two of eight Iraqi Jewish alleles 6 ; thus, it was added to the Iraqi mutation panel to increase the detection rate. The American College of Medical Genetics guidelines for the Jews of Ashkenazi origin established alternative criteria for carrier screening: a carrier frequency above 1:50 or a detection rate above $90 \% .^{13}$ Given that mutations common to Ashkenazi Jews were detected also in Oriental Jews, ${ }^{8}$ adding the five Ashkenazi mutations to the three founder Iraqi mutations, as recommended now by the ISMG, put the carrier frequency close to these criteria. However, further studies are required to establish the contribution of the five Ashkenazi mutations to the general Iraqi carrier pool.

The I1234V mutation had a carrier frequency of 1:130 (allele frequency $0.39 \%$ ) among Yemenite Jews screened in this study. Current available data put the CF carrier frequency in the Yemenite community below the ISMG inclusion criteria for carrier screening. Nevertheless, the I1234V mutation evaluation is offered when a Jewish Yemenite partner of a CF carrier is being evaluated, and can be added to the mutation panel of individuals of another screen-recommended origin whose background includes Yemenite.
This addition of the Iraqi and Yemenite Jewish groups to the carrier screening program will enable more precise estimation of residual risks for these groups, especially for intercommunal marriages in Israel. ${ }^{9}$

In recent months, CF carrier screening has been added to the already successful Tay Sachs screening covered by national health insurance in Israel. This shift in cost from private insurers and patients to the public health budget will reinforce the importance of CF screening and guarantee equal access for all.

Changes in the CF carrier screening protocol in Israel reflect its evolution from specific screening of founder mutations correlated to a specific ethnic group to expansion of the mutation panel and inclusion of more ethnic groups after detection of new mutations gleaned from population studies of screened individuals. The national screening policy takes into account the social trends of intercommunal admixture and the requirements for simplification of the screen while complying with allele frequency and mutation severity guidelines.

\section{REFERENCES}

1. Shanks H. Ancient Israel: a short history from Abraham to the Roman destruction of the temple. Englewood Cliffs, New Jersey: Prentice-Hall Biblical Archaeological Society, 1988.

2. Ostrer H. A genetic profile of contemporary Jewish populations. Nat Rev Genet 2001;2:891-897.

3. Kedar-Barnes I, Rozen P. The Jewish people: their ethnic history, genetic disorders and specific cancer susceptibility. Fam Cancer 2004;3:193-199.

4. Kerem E, Kalman YM, Yahav Y, et al. Highly variable incidence of cystic fibrosis and different mutation distribution among different Jewish ethnic groups in Israel. Hum Genet 1995;96:193-197.

5. Abeliovich D, Lavon IP, Lerer I, et al. Screening for five mutations detects $97 \%$ of cystic fibrosis (CF) chromosomes and predicts a carrier frequency of 1:29 in the Jewish Ashkenazi population. Am J Hum Genet 1992;51:951956

6. Quint A, Lerer I, Sagi M, Abeliovich D. Mutation spectrum in Jewish cystic fibrosis patients in Israel: implication to carrier screening. Am J Med Genet A 2005; 136:246-248.

7. Welsh MJ, Ramsey BW, Accurso FJ, Cutting GR. Cystic fibrosis. In: Scriver CR, Beaudet AL, Sly WS, Valle D, editors. The metabolic and molecular bases of inherited disease, 8th ed. New York: McGraw-Hill, 2001:51215188 .

8. Orgad S, Neumann S, Loewenthal R, Netanelov-Shapira I, Gazit E. Prevalence of cystic fibrosis mutations in Israeli Jews. Genet Test 2001;5: 47-52.

9. Cohen T, Vardi-Saliternik R, Friedlander Y. Consanguinity, intracommunity and intercommunity marriages in a population sample of Israeli Jews. Ann Hum Biol 2004;31:38-48.

10. Mussaffi H, Prais D, Mei-Zahav M, Blau H. Cystic fibrosis mutations with widely variable phenotype: the D1152H example. Pediatr Pulmonol 2006; 41:250-254

11. Grody WW, Cutting GR, Klinger KW, Richards CS, Watson MS, Desnick RJ. Laboratory standards and guidelines for population-based cystic fibrosis carrier screening. Genet Med 2001;3:149-154

12. Watson MS, Cutting GR, Desnick RJ. Cystic fibrosis population carrier screening: 2004 revision of American College of Medical Genetics mutation panel. Genet Med 2004;6:387-391.

13. Gross SJ, Pletche BA, Monaghan KG. ACMG practice guidelines: carrier screening in individuals of Ashkenazi Jewish descent. Genet Med 2008;10:54-56. 